\title{
First Results of Treatment of Leprosy with Rifadin $^{*+}$
}

\author{
D. L. LEIKER \\ Royal Tropical Institute, Amsterdam, and Dermatological Department, University of Amsterdam \\ H. KAMP \\ Q. M. Gastmann Wichers Foundation, Rotterdam
}

\begin{abstract}
The authors present the first published results obtained with Rifadin (rifampicin) in the treatment of 7 patients with lepromatous leprosy. The drug had a marked effect on the infecting bacilli. They conclude that Rifudin is an effective anti-leprotic drug which merits further and more extensive trials.
\end{abstract}

\section{INTRODUCTION}

Rifadin (Rifampicin) is a new "semi-synthetic" antibiotic, belonging to the group of rifamycins. Rifamycin S.V., isolated in 1957 from Streptomyces mediterranei n. sp., has shown antituberculosis and anti-leprosy properties (Opromolla, 1963; Merklen and Cottenot, 1963). Opromolla (1965) concluded that rifamycin is remarkably active clinically but the histopathological and bacteriological results do not always correspond with the clinical evaluation. The incidence of ENL reactions is moderate. No toxic effects have been found.

A disadvantage of rifamycin is that the drug has to be administered daily by injection. This is probably one of the reasons for the limited use of the drug in anti-leprosy treatment. The activity of rifadin against Mycobacterium tuberculosis is claimed to be even grater than that of rifamycin. Results of treatment of leprosy with this drug have not yet been reported. Rifadin has the great advantage that it can be administered orally in one dose daily.

Rifadin has shown activity in vitro and in vivo against various Gram-positive and some Gramnegative bacteria. It is inactive against fungi.

*Received for publication 1 January, 1970.

$\dagger$ The preliminary results of treatment of these patients were presented in a paper read at the Scientific Conference on Mycobacterial and Related Diseases, held at Dar es Salaam, Tanzania, in January, 1969.
In tuberculosis studies a very low toxicity has been found. In a series of 500 tuberculous patients treated with 450 to $600 \mathrm{mg}$ of rifadin daily, only one patient developed an allergic skin rash and a few patients complained of minor gastric distress; the gastric disturbances all occurred in patients receiving associated treatment with other drugs. In $15 \%$ of 150 tuberculosis patients drug resistance has been reported after treatment with rifadin for periods of 3 months or more.

In the present paper the first results of the treatment of 7 patients with lepromatous leprosy with rifadin are reported; 2 of these patients were clasified as "borderline lepromatous". The lepromin reaction in all the patients was negative with the exception of one borderline lepromatous patient who showed a Mitsuda reaction of $3 \mathrm{~mm}$. All the patients were strongly bacteriologically positive with a high percentage of non-granular bacilli. Treatment consisted in $600 \mathrm{mg}$ of rifadin daily, administered before breakfast in one dose. With one exception treatment was not interrupted during the reactive phases which occurred in some of the patients. The duration of treatment varied from 10 to 20 months.

\section{METHOD OF EVALUATION}

The patients were examined clinically at regular intervals and all reactions were recorded. The 
patients were asked to report any side-effects of the drug. Biopsy specimens were taken at regular intervals, in 6 cases at monthly intervals for the first 3 months of treatment and thereafter at 3-monthly intervals, and in the seventh patient at 3-monthly intervals only. The biopsy specimen was taken on each occasion from the same lesion, near to the site of the previous biopsies but avoiding the scars of these biopsies. The biopsy specimens were all processed in one laboratory (Amsterdam) and assessed by one assessor who at the time of examination had no information on the results of previous examinations. The bacteriological index (B.I.) (Ridley's scale), and the percentage of intact (solidly stained), of fragmented (somewhat unevenly stained, but not yet granular), and of granular bacilli was calculated.

\section{CASE HISTORIES}

\section{Patient No. l (R)}

Male, aged 23, West Indian, had borderline lepromatous leprosy with onset in 1953. He received irregular treatment with DDS between 1953 and 1967. Relapse in 1967 was followed by one month of treatment with thiambutosine. Rifadin treatment was started in October, 1967. At that time the patient showed multiple erythematous, moderately raised infiltrates on the face, trunk and extremities. Clinically and histopathologically a case of borderline lepromatous leprosy. The patient was strongly bacteriologically positive. Globi were present, and $59 \%$ of the bacilli were non-granular. After a few months of treatment the erythema had disappeared and the lesions had become flat. By March, 1968, the lesions were hardly still visible. During the 19 months of treatment no reactions had occurred and no side-effects had been observed. At the end of July, 1969, however, the patient complained of pain in the right arm, rapidly followed by muscle weakness. The right ulnar nerve was enlarged and painful. Rifadin was withdrawn and prednisone was administered. The neuritis rapidly subsided and one month later the paresis of the hand had disappeared.
Patient No. 2 (Sl)

Male, aged 25, West Indian, with lepromatous leprosy. Onset in 1966. He had had one month of treatment with $100 \mathrm{mg}$ of DDS weekly. Treatment with rifadin was started in February, 1968. at which time numerous erythematous nodules and small plaques were present on the face, trunk, and extremities. Clinically and histologically the patient was classified as having non-diffuse lepromatous leprosy, bacteriologically strongly positive with globi and $53 \%$ non-granular bacilli. After one month of treatment the lesions had become less erythematous and less raised, while after 3 months they had further subsided and had become hyperpigmented; one month later they were nearly flat. In July, 1968, however, the lesions suddenly became erythematous and raised again, though no new lesions appeared. The patient had no fever and no other complaints. The biopsy specimen showed no intact bacilli nor any decrease in the percentage of granular bacilli. The pseudo-exacerbation subsided spontaneously within one month, but 6 months later a similar pseudo-exacerbation was seen; however this also subsided rapidly and spontaneously. No other reactive phases have occurred during 20 months of treatment, and no other side-effects have been noticed.

\section{Patient No. $3(T j)$}

Male, age 34, West Indian, with lepromatous leprosy. Onset in 1954. Between 1954 and 1959 irregular DDS treatment. Relapse in 1965. Treatment with DDS was continued but often was interrupted by repeated $\mathrm{ENL}$ reactions. Rifadin treatment started in February, 1968.

At that time patient showed vague, diffuse infiltration of face and trunk and multiple nodular lesions on the extremities, in particular on the thighs and buttocks. The lesions were slightly erythematous. Clinicially and histologically the patient was classified as non-diffuse lepromatous. The patient was strongly bacteriologically positive, with globi and $67 \%$ nongranular bacilli. After one month of treatment the erythema had disappeared. After 3 months of 
treatment the lesions had become hyperpigmented and nearly flat. After 9 months of treatment only slight residual hyperpigmentation was visible. No reactions occurred during 12 months of treatment. No side effects have been noticed. Because patient decided to leave the country rifadin treatment was replaced by thiambutosine.

Patient No. $4(B)$

Male, age 30, West Indian, also had lepromatous disease, with onset in 1962. Between 1963 and 1967 he had had irregular treatment with DDS with repeated reactions. Treatment with rifadin was started in February, 1968. At that time the patient showed the picture of advanced, active, nodular lepromatous leprosy. In addition to extensive diffuse infiltrations and numerous firm erythematous cutaneous nodules, many deeply located nodules and plaques were also present. The lower legs were markedly swollen, indurated and painful. Smears were strongly bacteriologically positive, with globi and $63 \%$ non-granular bacilli. This was clinically and histologically a case of non-diffuse lepromatous leprosy.

In the first 2 months of treatment the erythema disappeared and the lesions subsided slightly. One mild erythema nodosum leprosum (ENL) reaction occurred. The patient com: plained of severe pains in his lower legs and feet, but refused further treatment because he thought that the drug aggravated the pain in his legs. One month later, however, he agreed to continue treatment again and in the next 6 months the lesions slowly subsided. During this period he had 3 mild to moderately severe ENL reactions and one attack of iridocyclitis. Although the patient still complained of pain in his legs, the induration of the legs gradually became less. In November, 1968, after 9 months of treatment, many deeply located nodules became painful and some more superficially located nodules became erythematous and a few ENL nodules appeared. The patient had fever and felt very ill. The reaction was treated with thalidomide and subsided within one week. During the following months the induration of the legs became markedly less. One mild and one moderately severe reaction, with painful deep nodules but without ENL lesions, occurred. The other infiltrates, although still conspicuous, had subsided markedly. During 19 months of treatment no other side-effects were observed. The condition of the patient has significantly improved.

\section{Patient No. $5\left(M W^{*}\right)$}

Female, age 45, Indo-European, lepromatous. Onset in 1964. Patient had had no previous treatment. Treatment with rifadin was started in October 1968. At that time slight vague, diffuse, symmetrical, copper-coloured but rather inconspicuous infiltrations were present. The face was puffy and the ear lobes were slightly swollen. Smears were strongly positive, with globi and $51 \%$ non-granular bacilli. The patient was classified as having diffuse lepromatous leprosy. After a few months of treatment with rifadin the erythema had disappeared and the infiltration had subsided. During 12 months of treatment no reactions occurred. No other side-effects have been seen and the clinical signs of leprosy have largely disappeared.

Patient No. 6 (M)

Male, age 33, West Indian, suffering from borderline lepromatous leprosy with onset in 1942. From 1942 to 1944 he had treatment with diasone, followed by very irregular treatment with DDS until 1965. In this period several exacerbations occurred. In 1968 the patient had a severe relapse, and rifadin treatment was started in October, 1968. At that time the patient showed numerous, nearly symmetrically distributed, fairly well-defined, reddish brown plaques and nodules on the face, trunk, and extremities. Some very well-defined, shiny nodules were present on the neck. The ears showed moderate infiltration. The eyebrows

\footnotetext{
* Serial biopsies of this patient have been received from Dr. A. H. Klokke of the Dermatological Department, University of Utrecht, whose kind co-operation we gratefully acknowledge.
} 
were only slightly thinned. Both hands showed moderate muscular atrophy and slight contractures of both fifth fingers. All major peripheral nerve trunks were markedly enlarged. The ulnar nerves were very thickened and painful. Bacteriologically, smears were strongly positive, with globi and $32 \%$ non-granular bacilli.

After one month of treatment with rifadin the skin lesions had become less erythematous and the nerve pain had subsided. After 3 months of treatment, however, the patient complained of pain in his neck, and the great auricular nerve was found to be very thickened and painful. He also complained of irradiating pains in the arms and legs. The neuritis subsided completely after 3 days of treatment with thalidomide. Similar attacks of neuritis were seen in February and March, 1969, but again quickly responded to thalidomide. In May another attack of neuritis was accompanied by fever and some ENL nodules. This reaction also responded rapidly to thalidomide. In July a mild reaction in the great auricular nerve occurred, but in the last 3 months no reactions have been seen. During the 12 months of treatment the skin lesions have subsided markedly.

\section{Patient No. $7(K)$}

Male, age 38, West Indian, with lepromatous leprosy. Onset in 1948. He had received irregular treatment with DDS between 1948 and 1966, but suffered an exacerbation in 1968. Tieatment with rifadin was started in December, 1968. At that time the patient presented the picture of advanced secondary nodular lepromatous leprosy. Smears were bacteriologically strongly positive, with globi and $35 \%$ non-granular bacilli. After 3 months of treatment the lesions showed signs of improvement; they became hyperpigmented and slightly wrinkled. In April, 1969 , the patient complained of pain in his right arm. The ulnar nerved was found to be markedly enlarged and slightly painful on palpation. In May he developed fever and a few ENL nodules were seen. The reactions responded quickly to treatment with thalidomide. In July the patient had another attack of fever, but without ENL nodules. No other cause was found. The reaction again responded rapidly to thalidomide. A similar reaction was seen in August. During the 10 months of treatment with rifadin the skin lesions have markedly subsided and have become strongly hyperpigmented. No other side-effects have been noted.

\section{BACTERIOLOGICAL EVALUATION}

In all the cases here described some increase in the percentage of granular bacilli was seen within one month of starting treatment. In 5 out of the 7 patients (see Table 1 ) after 3 months of treatment the great majority of the bacilli had become granular. In one of the patients who had stopped treatment for one month a delay was seen, but the percentage of granular bacilli increased rapidly again soon after treatment had been resumed. In another patient, however, it took almost 9 months before

TABLE I

Bacteriological progress in 7 patients treated with rifadin, showing percentage of bacilli intact (I), fragmented (F), and granular (G)

\begin{tabular}{|c|c|c|c|c|c|c|c|c|c|c|c|c|c|c|c|c|c|c|c|c|c|c|c|c|c|c|c|c|}
\hline \multirow[t]{2}{*}{ Patient number } & \multicolumn{4}{|c|}{$\begin{array}{l}1 \\
\text { Percentage }\end{array}$} & \multicolumn{4}{|c|}{$\stackrel{2}{\text { Percentage }}$} & \multicolumn{4}{|c|}{$\stackrel{3}{\text { Percentage }}$} & \multicolumn{4}{|c|}{$\stackrel{4}{\text { Percentage }}$} & \multicolumn{4}{|c|}{$\begin{array}{c}5 \\
\text { Percentage }\end{array}$} & \multicolumn{4}{|c|}{$\begin{array}{c}6 \\
\text { Percentage }\end{array}$} & \multicolumn{4}{|c|}{$\begin{array}{l}7 \\
\text { Percentage }\end{array}$} \\
\hline & B.I. & 1 & $F$ & G & B.I. & 1 & $F$ & $G$ & B.I. & 1 & $F$ & $G$ & B.I. & 1 & $F$ & G & B.I. & 1 & $F$ & $G$ & B.I. & 1 & $F$ & G & B.I. & 1 & $F$ & G \\
\hline Onset & 5.5 & 15 & 44 & 41 & 6.0 & 3 & 50 & 47 & 6.0 & 6 & 61 & 33 & 6.0 & 9 & 54 & 37 & 5.0 & 1 & 50 & 49 & 4.0 & 0 & 32 & 68 & 6.0 & 2 & 33 & 65 \\
\hline I month & 5.0 & 2 & 20 & 78 & 5.0 & 1 & 44 & 55 & 5.0 & 0 & 17 & 83 & 6.0 & 0 & 20 & 80 & 5.0 & 0 & 33 & 67 & 4.0 & 0 & 0 & 100 & 6.0 & 0 & 3 & 97 \\
\hline 2 months & 5.0 & 0 & 16 & 84 & 4.5 & 0 & 15 & 85 & 5.0 & 0 & 9 & 91 & 6.0 & 0 & 30 & 70 & 5.0 & 0 & 17 & 83 & & & & & 6.0 & 0 & 3 & 97 \\
\hline 3 months & 4.5 & 0 & 14 & 86 & 5.0 & 0 & 1 & 99 & 5.0 & 0 & 1 & 99 & 5.0 & 0 & 15 & 85 & 5.0 & 0 & 0 & 100 & & & & & 6.0 & 0 & 4 & 96 \\
\hline 6 months & 4.5 & 0 & 13 & 87 & 6.0 & 0 & 1 & 99 & 4.0 & 0 & 0 & 100 & 4.0 & 0 & 0 & 100 & 5.0 & 0 & 2 & 98 & 4.0 & 0 & 0 & 100 & 6.0 & 0 & 6 & 94 \\
\hline 9 months & 4.0 & 0 & 1 & 99 & 4.0 & 0 & 0 & 100 & 6.0 & 0 & 0 & 100 & 6.0 & 0 & 1 & 99 & 5.0 & 0 & 6 & 94 & 6.0 & 0 & I & 99 & 6.0 & 0 & 1 & 99 \\
\hline 12 months & 3.5 & 0 & 0 & 100 & 6.0 & 0 & 3 & 97 & 6.0 & 0 & 3 & 97 & 6.0 & 0 & 0 & 100 & 6.0 & 0 & 1 & 99 & 6.0 & 0 & 0 & 100 & 5.5 & 0 & 0 & 100 \\
\hline 15 months & 3.0 & 0 & 0 & 100 & 5.0 & 0 & 0 & 100 & & & & & 6.0 & 0 & 2 & 98 & & & & & & & & & & & & \\
\hline 18 months & 4.0 & 0 & 6 & 94 & 3.0 & 0 & 1 & 99 & & & & & 4.0 & 0 & 3 & 97 & & & & & & & & & & & & \\
\hline
\end{tabular}


most of the bacilli had become granular; no explanation can be offered for the delay in this case. In conclusion, the decrease in the morphological index in patients receiving treatment with rifadin is at least as rapid as in those treated with sulphones and other anti-leprosy drugs. In the first 6 months of treatment a satisfactory decrease in the Bacterial Index (B.I.) was seen in several of the patients. The average B.I. did not decrease significantly in the second halfyear. In 3 of the patients an increase in the B.I. occurred. These findings could be explained by the rapid clinical response to rifadin. In most patients the size of the lesions substantially diminished, resulting in concentration of the bacilli. The possibility of drug-resistance has also been considered. In none of the patients, however, have intact bacilli reappeared, nor has the percentage of granular bacilli increased significantly, nor have any clinical signs of relapse been found. Histopathologically no evidence of relapse has been seen.

\section{SIDE-EFFECTS}

In 5 of the 7 patients reactions of some kind were recorded. In most cases the reactions were mild to moderately severe. In all but one patient (No. 1) treatment with rifadin was continued during these reactions. No evidence that continuation of rifadin treatment during reactions had an aggravating effect on the reactions was found. No other side-effects of the drug were observed.

\section{DISCUSSION}

Usually the bacteriological status of a patient is given by the morphological index, which is the percentage of solidly stained, presumably viable bacilli. All other bacilli are regarded as non-viable. Our method of assessment is different. In our opinion it is of ten difficult to distinguish with certainty between completely and evenly stained bacilli (intact) and bacilli which are slightly changed (fragmented) but not yet granular. Therefore 3 instead of 2 percentages are given. We regard the intact bacilli as definitely viable. Since one of us (D).L.) has obtained growth of Myco. leprae in the footpads of mice from material taken from a patient with $6 \%$ fragmented and $\mathbf{9 4} \%$ granular bacilli, a proportion of the fragmented bacilli must have been viable. A high percentage of our fragmented bacilli would be included by other workers in the group of solid bacilli. The percentage of viable bacilli in all our patients in this study was therefore high at the onset of the trial. The most spectacular observation in this trial was the very rapid decline in the percentage of viable bacilli in most of the patients, more rapid than has been seen in patients treated with other anti-leprosy drugs. The manufacturers claim that rifadin is a bacteriocidal drug. The findings in this trial confirm this statement. The elimination of dead bacilli in the first year of treatment with rifadin seems to be slow. After treatment with other drugs the average annual fall in the B.I. is about $1+$. There was, however, a rapid reduction of the degree of infiltration, resulting in a concentration of bacilli in the lesions. Besides, all these patients were very highly bacilliferous. As $6+$ is the maximum in our scale, meaning 1000 or more bacilli per microscope field, and as the number of bacilli in our patients was far above 1000 per field, a possible reduction in the number of bacilli could not be accurately measured. Probably the rate of elimination of dead bacilli in patients treated with rifadin does not differ from those treated with other anti-leprosy drugs.

It is concluded that rifadin is an effective antileprosy drug meriting further investigation, especially in respect of the incidence and severity of reactions and of the development of drug resistance.

\section{SUMMARY}

A first report on the treatment of 7 lepromatous patients with rifadin, a new antibiotic with antituberculosis properties, related to rifamycin S.V. Significant clinical improvement was seen in all patients after administration of $600 \mathrm{mg}$ of rifadin daily for periods of between 12 and 20 months. In all cases some increase in the 
percentage of granular bacilli was seen already after one month. In all patients this percentage increased rapidly in the months that followed. In one patient, however, it took almost 9 months before nearly $100 \%$ of the bacilli had become granular. Although a satisfactory decrease in the average B.I. was seen after 6 months of treatment, in the second half-year no further average decrease was seen; this could be explained as being due to a greater concentration of bacilli in lesions which had rapidly subsided. In none of the patients has conclusive evidence of drug-resistance been found so far. In 5 patients reactions occurred; these were mild to moderately severe and rifadin treatment was continued during the reactions. There was no evidence that the reactions were aggravated by rifadin. No other side-effects were observed. It is concluded that rifadin is an effective antileprosy drug which merits further investigations, especially with regard to the incidence of reactions and the development of drug resistance.

\section{ACKNOWLEDGEMENT}

Rifadin has been generously supplied for this trial by the manufacturers: Messrs. Lepetit, Milan.

\section{REFERENCES}

MERklen, F. P. and cotTenot, F. (1963). Essai d'un nouvel antibiotique, la rifamycine S.V., dans quelques cas de maladie de Hansen. Bull. Soc. fr. Derm. Syph. 70, 528.

opromolla, D. v. A. (1963). First results of the use of rifamycin S.V. in the treatment of lepromatous leprosy. Eighth International Congress on Leprosy, Rio de Janeiro, 1963. Abstract in Int. .J. Lepr. 31, 552.

OPROMOLLA, D. V. A., DE SOUZA LimA, L. and CAPRARA, C. (1965). Rifamycin S.V. in the treatment of lepromatous leprosy. Lepr. Rev. 36, 123.

RIDLEY, D. S. (1964). Bacterial Indices. In Leprosy in Theory and Practice, 2nd ed., p. 620. Appendix III Bristol: John Wright. 